\title{
AN INFRARED COMPARISON OF TYPE-1 AND TYPE-2 QUASARS
}

\author{
Kyle D. Hiner ${ }^{1}$, Gabriela Canalizo ${ }^{1}$, Mark Lacy ${ }^{2}$, Anna Sajina ${ }^{3}$, Lee Armus ${ }^{2}$, Susan Ridgway ${ }^{4}$, \\ AND Lisa STORRIE-LOMBARDI ${ }^{2}$ \\ ${ }^{1}$ Department of Physics and Astronomy \& Institute of Geophysics and Planetary Physics, University of California, Riverside, CA 92521, USA; \\ kyle.hiner@email.ucr.edu, gabriela.canalizo@ucr.edu \\ ${ }^{2}$ Spitzer Science Center, California Institute of Technology, Pasadena, CA 91125, USA; mlacy@ipac.caltech.edu, lee@ipac.caltech.edu, lisa@ipac.caltech.edu \\ ${ }^{3}$ Department of Physics \& Astronomy, Haverford College, Haverford, PA 19041, USA; asajina@ haverford.edu \\ ${ }^{4}$ NOAO/CTIO, 950 N. Cherry Ave, Tuscon, AZ 85719, USA; seridgway@ctio.noao.edu \\ Received 2009 March 18; accepted 2009 October 7; published 2009 November 2
}

\begin{abstract}
We model the optical to far-infrared spectral energy distributions (SEDs) of a sample of six type-1 and six type-2 quasars selected in the mid-infrared. The objects in our sample are matched in mid-IR luminosity and selected based on their Spitzer IRAC colors. We obtained new targeted Spitzer Infrared Spectrograph and Multiband Imaging Photometer for Spitzer observations and used archival photometry to examine the optical to far-IR SEDs. We investigate whether the observed differences between samples are consistent with orientation-based unification schemes. The type-1 objects show significant emission at $3 \mu \mathrm{m}$. They do not show strong polycyclic aromatic hydrocarbon (PAH) emission and have less far-IR emission on average when compared to the type- 2 objects. The SEDs of the type-2 objects show a wide assortment of silicate features, ranging from weak emission to deep silicate absorption. Some also show strong PAH features. In comparison, silicate is only seen in emission in the type-1 objects. This is consistent with some of the type-2's being reddened by a foreground screen of cooler dust, perhaps in the host galaxy itself. We investigate the active galactic nucleus contribution to the far-IR emission and find it to be significant. We also estimate the star formation rate (SFR) for each of the objects by integrating the modeled far-IR flux and compare this with the SFR found from PAH emission. We find that the type- 2 quasars have a higher average SFR than the type-1 quasars based on both methods, though this could be due to differences in bolometric luminosities of the objects. While we find pronounced differences between the two types of objects, none of them are inconsistent with orientation-based unification schemes.
\end{abstract}

Key words: infrared: galaxies - quasars: general

\section{INTRODUCTION}

The study of various types of active galactic nuclei (AGNs) can provide insight into their origin and evolution. AGNs are classified according to their optical emission lines as type-1 or type-2. Type-1 AGNs have an "unobscured" view of the hot accretion disk and broad emission lines originating from fast moving clouds that surround a supermassive black hole. Type-2 objects are known as "obscured" AGNs, because no broad lines or continuum emission from the accretion disk are observed, yet the spectra still show narrow emission lines similar to those of the type- 1 objects.

The interpretation of the differences between type- 1 and type-2 quasars (the most luminous AGNs) remains an open issue. The orientation-based unification scheme proposes that the two types of objects are intrinsically the same, but appear different observationally due to the presence of an obscuring torus of dust close to the central power source (see Antonucci 1993). Type-1 objects are seen from an orientation which allows an unobscured view of the central source. This allows the broad line region of the quasar to be observed. However, type- 2 objects are seen through the dusty torus material, which hides the central continuum source and broad-line region. Evidence that this model is at least partly correct comes from observations of polarized broad-line emission in galaxies classified as type-2 quasars (e.g., Zakamska et al. 2005). But this model does not provide insight into the origin of quasar activity.

An alternative model proposes that quasar activity is triggered by galactic mergers and evolves through an obscured stage (Sanders et al. 1988). As the galaxies merge, gas and dust are stirred up, triggering quasar activity and star formation.
Initially, the quasar would be obscured by the dust, but over time will become unobscured as dust and gas are blown away from the nuclear region by quasar- and/or supernovae-driven winds and radiation pressure. Indeed, several host galaxy studies have been made which show that luminous AGNs are hosted by massive, mainly spheroid-dominated galaxies, with a significant fraction, but not all, showing signs of a relatively recent merger and associated star formation (e.g., Dunlop et al. 2003; Canalizo et al. 2007; Bennert et al. 2008). Thus, the evolution model predicts that the host galaxies of type- 2 quasars should have more recent star-forming activity than those of type-1's. Although the discovery of polarized broad lines is a powerful argument in favor of the orientation-based model, it does not rule out an evolutionary link.

The obscuring dust in a host galaxy would be apparent in its observed spectral energy distribution (SED). Dust close to the AGN would be heated by the central power source and would thermally re-radiate in the mid-IR $(100-1000 \mathrm{~K})$, dominating the rest-frame SED from $\sim 2-40 \mu \mathrm{m}$. This high-temperature dust emission makes the mid-IR SEDs of both optically obscured and optically unobscured AGNs quite distinct from those of either normal or starburst galaxies, whose dust temperatures are always $\leqslant 100 \mathrm{~K}$. By modeling the various components of the SEDs, we can compare the relative amounts of "cool", "warm", and "hot" dust emission present in the host galaxies. This will give us some idea of what mechanism dominates the heating of the dust (star formation or AGN), and how much of a role orientation might play in the observed SEDs.

In this paper, we present modeled fits to the SEDs of a sample of type-1 and type- 2 quasars of similar redshift and luminosity. Lacy et al. (2007) presented preliminary fits to the 
Table 1

Targets and Their Fluxes in mJy

\begin{tabular}{|c|c|c|c|c|c|c|c|c|}
\hline Target & Type & $z$ & $f_{24}$ & $\sigma_{24}$ & $f_{70}$ & $\sigma_{70}$ & $f_{160}$ & $\sigma_{160}$ \\
\hline SDSS 171126.94+585544.2 & 1 & 0.537 & 3.45 & 0.07 & 12.7 & 6.6 & $<102.1$ & $\cdots$ \\
\hline SDSS $171736.91+593011.5$ & 1 & 0.599 & 6.38 & 0.05 & 12.9 & 2.5 & $<15.9$ & 5.3 \\
\hline SDSS $171748.43+594820.6$ & 1 & 0.763 & 3.04 & 0.04 & 9.7 & 4.1 & $<9.6$ & 3.2 \\
\hline SDSS $171818.14+584905.2$ & 1 & 0.634 & 4.06 & 0.06 & 35.0 & 6.1 & 28.9 & 13.9 \\
\hline SST $171147.4+585839$ & 2 & 0.800 & 4.84 & 0.07 & 18.8 & 5.7 & 31.9 & 13.7 \\
\hline SST $171324.1+585549$ & 2 & 0.609 & 4.94 & 0.07 & 16.1 & 1.5 & 33.8 & 10.7 \\
\hline SST $171831.7+595317$ & 2 & 0.700 & 8.27 & 0.04 & 27.1 & 4.4 & 31.2 & 12.8 \\
\hline SST $172123.1+601214$ & 2 & 0.325 & 13.34 & 0.07 & 12.0 & 6.2 & $<9.3$ & 3.1 \\
\hline SST $172458.3+591545$ & 2 & 0.494 & 2.60 & 0.06 & 24.6 & 4.9 & 38.1 & 15.0 \\
\hline
\end{tabular}

type-2 quasars in this paper. We also measure the objects' far-IR luminosities, polycyclic aromatic hydrocarbon (PAH) luminosities, and estimate their star formation rates (SFR). We then compare the results with starburst galaxies. We describe the sample selection in Section 2, and the observations and reductions in Section 3. In Section 4, we present the analysis and results including our modeling procedure and resultant spectra, and SFR calculations. Finally, we summarize our results in Section 5. Throughout this paper, we adopt the cosmological parameters of $H_{0}=71 \mathrm{~km} \mathrm{~s}^{-1} \mathrm{Mpc}^{-1}, \Omega_{M}=0.27$, and $\Omega_{\Lambda}=0.73$.

\section{SAMPLE SELECTION}

Quasar surveys are now highly effective at finding quasars on the basis of their optical colors, provided they are not reddened or only lightly reddened by dust. With surveys such as the Sloan Digital Sky Survey (SDSS; Schneider et al. 2003), 2dF (Croom et al. 2004), and Combo-17 (Wolf et al. 2003), a census of such objects across the full range of quasar luminosities and redshifts out to $z \sim 6$ is largely complete. Only recently have radio-quiet type- 2 quasars been found in substantial numbers. However, there is still no consensus on how the dust-obscured quasars are related to the unobscured population. In particular, it is difficult to select matched samples of type-1 and type-2 objects with which to perform such studies. By using Spitzer data from the extragalactic First Look Survey (xFLS), we have been able to construct samples of type-1 and type-2 quasars matched in mid-IR luminosity and redshift.

Lacy et al. (2004) showed that quasars occupy a distinct "sequence" of a Spitzer IRAC color-color plot (their Figure 1). Follow-up spectroscopy of a sample of mid-IR selected AGN and quasar candidates showed that about $2 / 3$ of AGN selected in this manner may be sufficiently obscured by dust in the optical to be missing from optically selected quasar samples (Lacy et al. 2007). We selected six type-2 quasars and six type1 quasars (see Table 1) from the $1 \mathrm{mJy} 8 \mu \mathrm{m}$ flux-limited sample of Lacy et al. (2004), with similar mid-IR colors and $5.8 \mu \mathrm{m}$ luminosities. These objects are approximately matched in midIR luminosity. Our modeling (see Section 4.1) shows they have mid-IR power-law luminosities within a factor of 5 from each other. Furthermore, the observed frame $24 \mu \mathrm{m}$ luminosities of the type- 1 and type- 2 quasars are not statistically different from each other. It is important that the sample be matched in luminosity, since the quasar luminosity will affect the rate at which dust and gas are removed from the nuclear region.

\section{OBSERVATIONS AND DATA REDUCTION}

We supplement data from the Spitzer Extragalactic First Look Survey (xFLS; Fadda et al. 2006; Frayer et al. 2006) with targeted observations in the $70 \mu \mathrm{m}$ and $160 \mu \mathrm{m}$ using the Multiband Imaging Photometer for Spitzer (MIPS). Each of our targets was detected at the $24 \mu \mathrm{m}$ wavelength in the Spitzer $\mathrm{xFLS}$. Five of our targets were detected in the $70 \mu \mathrm{m}$ xFLS mosaic, and we measured the $70 \mu \mathrm{m}$ fluxes for two other targets directly from the xFLS mosaic image. The remaining five targets of our sample were not detected in the $70 \mu \mathrm{m}$ xFLS mosaic and so required targeted observations. We observed each of these targets for $500 \mathrm{~s}$. All of the targets were observed with the MIPS $160 \mu \mathrm{m}$ band for $2000 \mathrm{~s}$ each.

The photometric data were reduced through the standard Spitzer MIPS pipeline. We used the post-basic calibrated (post$\mathrm{BCD}$ ) filtered mosaic images to measure the flux of each target. The pixel scales of the post-BCD MIPS images are 4 arcsec pixel ${ }^{-1}$ at $70 \mu \mathrm{m}$ and 8 arcsec pixel ${ }^{-1}$ at $160 \mu \mathrm{m}$.

We also observed each target using the short-low and long-low resolution modes with the Spitzer infrared spectrograph (IRS). The spectra were reduced through the standard Spitzer pipeline. We manually masked the bad pixels in the background subtracted spectra (difference between nodded exposure positions) using the IDL program IRSCLEAN. ${ }^{5}$ We then extracted the spectra using the standard point-source extraction in the Spitzer SPICE $^{6}$ software tool, and combined the individual spectra to create a continuous mid-IR spectrum for each target. Lastly, we included photometry measured from our MIPS data (Table 1) and data available for our targets from SDSS, Two Micron All Sky Survey (2MASS), and Spitzer IRAC observations (Table 2) to produce an observed frame SED that ranges from optical through the infrared.

\subsection{Photometry}

We used the DAOPHOT package in IRAF to measure the $70 \mu \mathrm{m}$ and $160 \mu \mathrm{m}$ fluxes of our targets. For the $70 \mu \mathrm{m}$ measurements, we examined each image individually and determined the best aperture and sky annulus that maximized the signal-tonoise ratio $(\mathrm{S} / \mathrm{N})$. We then applied aperture corrections from the Spitzer Space Telescope MIPS Data Handbook, ${ }^{7}$ and compared the measured flux to a $3 \sigma$ limit obtained by measuring the noise in the image.

\footnotetext{
5 http://ssc.spitzer.caltech.edu/archanaly/contributed/irsclean

6 http://ssc.spitzer.caltech.edu/postbcd/spice.html

7 http://ssc.spitzer.caltech.edu/mips/apercorr/
} 
Table 2

Additional Photometry Used in Modeling Procedure

\begin{tabular}{|c|c|c|c|c|c|c|c|c|c|c|}
\hline Target & $u$ & $g$ & $r$ & $i$ & $z$ & $J$ & $H$ & $K$ & $S_{3.6}$ & $S_{4.5}$ \\
\hline SDSS $171117.66+584123.8$ & 19.23 & 18.70 & 18.61 & 18.33 & 18.37 & 17.04 & 16.10 & 15.63 & 1.26 & 1.61 \\
\hline SDSS $171126.94+585544.2$ & 19.27 & 18.86 & 18.94 & 18.75 & 18.70 & $\ldots$ & $\cdots$ & $\ldots$ & 0.64 & 0.81 \\
\hline SDSS $171736.91+593011.5$ & 19.36 & 18.85 & 18.92 & 18.72 & 18.82 & 18.53 & $\cdots$ & 16.19 & 0.46 & 0.61 \\
\hline SDSS $171748.43+594820.6$ & 18.42 & 18.06 & 18.07 & 18.14 & 18.09 & 18.04 & $\cdots$ & 16.05 & 0.65 & 0.90 \\
\hline SDSS $171818.14+584905.2$ & 19.17 & 18.70 & 18.70 & 18.57 & 18.70 & $\cdots$ & $\cdots$ & $\cdots$ & 0.68 & 0.87 \\
\hline SST $171147.4+585839$ & 22.94 & 22.30 & 21.61 & 20.80 & 20.31 & 18.8 & 18.0 & 16.6 & 0.52 & 0.82 \\
\hline SST 171324.1+585549 & 22.82 & 21.83 & 20.95 & 20.21 & 20.06 & 19.0 & 18.2 & 17.3 & 0.20 & 0.43 \\
\hline SST $171831.7+595317$ & 22.93 & 21.91 & 20.93 & 20.18 & 19.78 & 18.7 & 18.0 & 17.2 & 0.16 & 0.26 \\
\hline SST $172123.1+601214$ & 21.69 & 20.28 & 18.99 & 18.74 & 18.33 & 17.8 & 16.5 & 15.2 & 1.03 & 1.60 \\
\hline SST $172458.3+591545$ & 21.94 & 21.69 & 20.31 & 19.61 & 19.17 & 18.1 & 17.2 & 16.3 & 0.43 & 0.49 \\
\hline
\end{tabular}

Notes. Additional photometry for the modeling procedure was retrieved from NASA Extragalactic Database (NED). SDSS $u, g, r, i$, and $z$ magnitudes are listed in the AB system (Oke 1990). The $u$ and $g$ photometry were not used in the fitting of the type- 2 targets. 2MASS $J, H$, and $K_{s}$ magnitudes are presented in Vega system. IRAC bands 1 and $2(3.6 \mu \mathrm{m}$ and $4.5 \mu \mathrm{m})$ are listed in mJy.

Each of our 12 targets was observed in the $160 \mu \mathrm{m}$ MIPS band, though many of our targets were not detected at this wavelength. We chose an example image with a strong detection and determined the aperture and sky annulus sizes that maximized the $\mathrm{S} / \mathrm{N}$. We used those parameters while measuring the other images with bright detections. The same procedure was used for the images with targets that were not detected. We then compared the measured fluxes of each of the targets to the $3 \sigma$ limit determined from the noise in the image. Five of the 12 targets have fluxes less than the $2 \sigma$ noise limit, and we count them as non-detections. Five other targets have fluxes $>2 \sigma$, and only one has $160 \mu \mathrm{m}$ flux $>3 \sigma$. The remaining target is discussed in the following.

The target SDSS $1711+5855$ required special attention, because there was a bright object near the target. We fit a pointspread function (PSF) using several other bright objects in the image, then subtracted sources from the image leaving only our target. We then measured the flux of SDSS 1711+5855 from the subtracted image. This procedure was successful at $70 \mu \mathrm{m}$, but our attempts at removing the extra source at $160 \mu \mathrm{m}$ resulted in an image with significant residuals. Therefore, we only report an upper limit measured from the combined flux of the objects at $160 \mu \mathrm{m}$. This upper limit was not used in our modeling procedure and analysis.

Table 1 shows the results of our MIPS flux measurements combined with data from the xFLS.

\subsection{Spectra}

We present the Spitzer IRS spectra for our sample in Figure 1. The spectra show distinct differences. The spectra of the type-1 quasars tend to have fewer features and are more homogeneous than those of the type-2's. The type-1 objects show significant continuum emission near $3 \mu \mathrm{m}$, little PAH emission, and no significant silicate absorption. The type-2 spectra are more heterogeneous, showing a variety of silicate absorption depths and PAH strengths, consistent with previous observations of AGNs (e.g., Hao et al. 2007; Spoon et al. 2007; Zakamska et al. 2008) as well as starburst galaxies, ULIRGs and submillimeter galaxies (e.g., Brandl et al. 2006; Sajina et al. 2007; Lutz et al. 2008; Menendez-Delmestre et al. 2009). The type-2 quasars have on average double the PAH luminosity of the type- 1 quasars (see Table 3).

Spoon et al. (2007) classify spectra based on their $6.2 \mu \mathrm{m}$ PAH equivalent width and $9.7 \mu \mathrm{m}$ silicate absorption strength. Our type-2 spectra are similar to their $1 \mathrm{~B}, 1 \mathrm{C}$, and $2 \mathrm{~B}$ classifications. These classifications are dominated by Seyferts, starbursts, and ULIRGs, respectively. Our type-1 quasars have spectra most similar to the 1A classification of galaxies by Spoon et al.

The variation of silicate absorption strengths in the type2 spectra of our sample is also consistent with that seen by Zakamska et al. (2008), who study a sample of optically selected type-2 quasars from the SDSS. Zakamska et al. find silicate absorption similar to some of our sample, including two objects that have more significant absorption than our sample (see Lacy et al. 2007). Four of their objects appear to have no silicate absorption, including one that may show silicate emission, consistent with our mid-IR-selected type-1 quasars. They also address the correlation of silicate absorption with axial ratio found by Lacy et al. (2007). Lacy et al. interpreted this correlation to indicate that the silicate dust resides in the host galaxy, and not the nuclear region. The objects of Zakamska et al. (2008) show no correlation, and they propose this could be a difference in samples due to selection biases based on luminosity.

\section{ANALYSIS AND RESULTS}

\subsection{Modeling}

We modeled the SEDs of our sample from the optical out to the far-IR. We used available SDSS and 2MASS photometry, IRAC bands 1 and $2(3.6 \mu \mathrm{m}$ and $4.5 \mu \mathrm{m}$, respectively) photometry (Lacy et al. 2005) and our Spitzer IRS mid-IR spectra and MIPS photometry. This additional photometry used in the modeling procedure is presented in Table 2 . The modeling code is described by Sajina et al. (2006). Modifications to the code to enable modeling of the type- 2 objects in our sample were briefly described by Lacy et al. (2007). Here, we provide a fuller description of the modeling, and the additional modifications required to fit the type- 1 objects. The modeling is phenomenological in nature due to the current lack of a good physical understanding of the nature and geometry of the dust emitting regions, particularly in the mid-IR, thus many of the parameters do not have a direct physical interpretation. Nevertheless, we considered the effort worthwhile as it allows an empirical breakdown of the contributions of starlight, hot dust emission from the AGN, and cooler dust emission.

The type-2 SEDs were modeled using the following components. (1) A single stellar population from Bruzual \& Charlot 

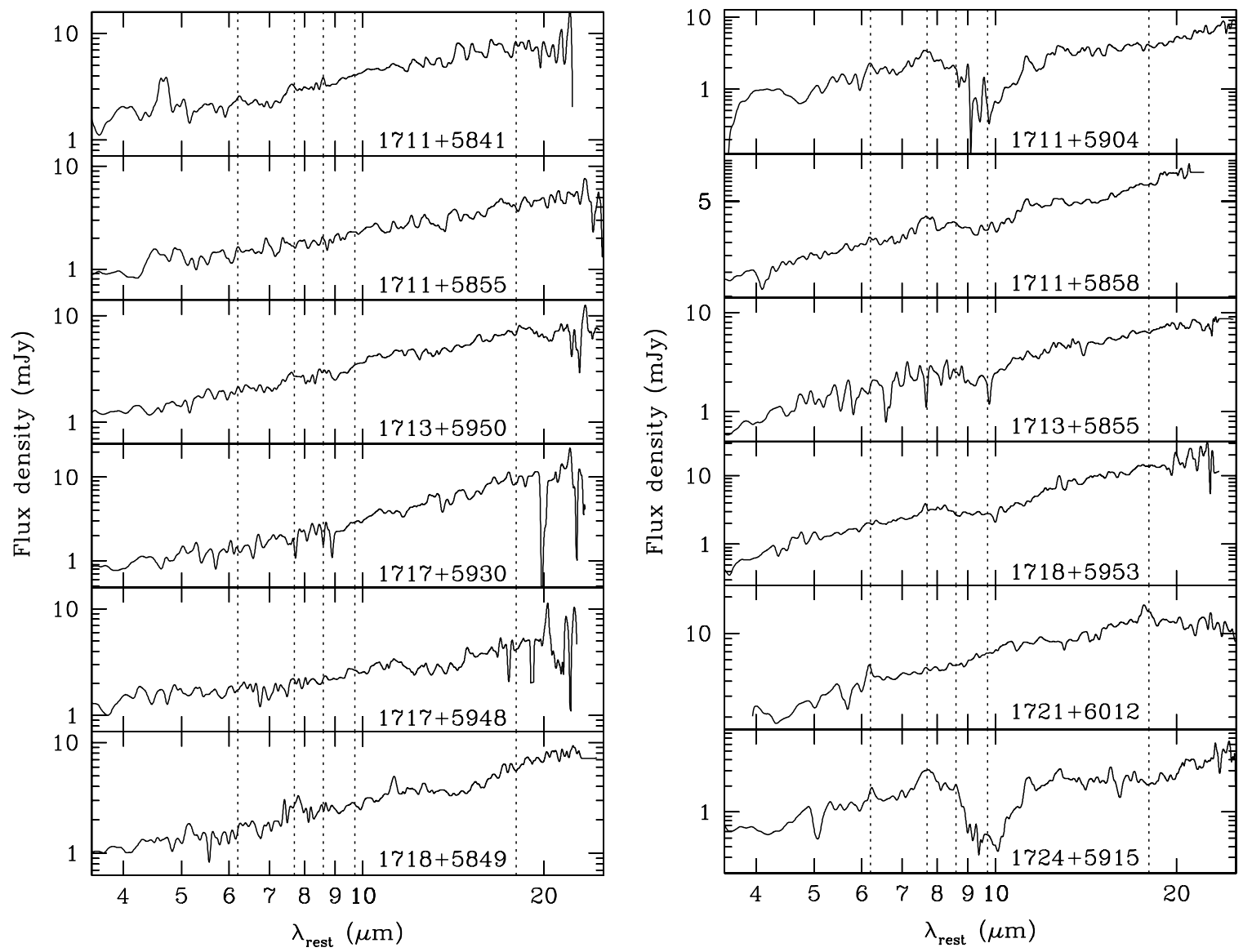

Figure 1. Left: IRS spectra for our type-1 quasar sample. Right: IRS spectra for our type-2 quasar sample. The spectra have been smoothed with a Gaussian with sigma of 5 pixels. Vertical dotted lines indicate the wavelengths for 6.2, 7.7, and $8.6 \mu \mathrm{m}$ PAH features, and 9.7 and $18 \mu \mathrm{m}$ silicate features.

(2003) with an age of 5 Gyr to represent the evolved stellar population. (2) A power-law component for the mid-IR emission, with an exponential cutoff at short wavelengths to represent dust sublimation, and a Fermi function cutoff at long wavelengths designed to match the SED shape of the least star-forming object in the sample of type-2's, SST 1721+6012. The functional form used was

$$
L_{\mathrm{AGN}}=\frac{L_{\mathrm{AGN}}^{0} \nu^{1-\alpha} e^{h \nu / k_{B} T_{\text {sub }}}}{e^{\left(v-\nu_{\text {hcut }} / w\right.}+1}
$$

where the normalization, $L_{\mathrm{AGN}}^{0}$, power-law index $\alpha$ and $T_{\text {sub }}$ (a proxy for the sublimation temperature) are allowed to vary, and $v_{\text {hcut }}$ and $w$ were fixed at $0.11 \times 10^{14} \mathrm{~Hz}$ and $0.017 \times 10^{14} \mathrm{~Hz}$, respectively ( $h$ and $k_{\mathrm{B}}$ are the Planck and Boltzman constants, respectively). The Galactic Center extinction curve of Chiar \& Tielens (2006) was then applied to this continuum to fit any silicate absorption feature. (3) A warm dust component to represent the small grain emission from $\mathrm{H}$ in regions, represented by a power law with fixed high and low frequency cutoffs:

$$
L_{\mathrm{SG}}=L_{\mathrm{SG}}^{0} v^{1-\gamma} e^{-\nu / \nu_{\mathrm{sgl}}} e^{-v_{\mathrm{sgh}} / \nu} .
$$

This component is poorly constrained in most of the fits, so $\gamma$ was fixed at a typical value of two (e.g. Sajina et al. 2006). The high and low frequency cutoffs, $v_{\text {sgl }}$ and $v_{\text {sgh }}$ were set to $0.062 \times 10^{14} \mathrm{~Hz}$ and $0.17 \times 10^{14} \mathrm{~Hz}$, respectively. (4) a thermal modified blackbody model for the far-infrared emission:

$$
L_{\mathrm{FIR}}=\frac{L_{\mathrm{FIR}}^{0} v^{3+\beta}}{e^{h \nu / k_{\mathrm{B}} T}-1} .
$$

For most objects, we fixed the temperature of this component, $T$, to $45 \mathrm{~K}$, because of the uncertainty in our $160 \mu \mathrm{m}$ photometry. $\beta$ was fixed at 1.5. In addition, the PAH model described in Lacy et al. (2007) was added when evidence of PAH emission was seen, and SST 1721+6012 had a silicate emission profile added, whose shape was approximated by that of the extinction curve.

For the type-1 quasars, component (1) was replaced by an SDSS composite quasar spectrum. As the composite of Vanden Berk et al. (2001) suffers from noticeable host galaxy contamination at the long wavelength end, we constructed a new composite by subtracting the continuum from the composite of Vanden Berk et al., and adding the residual emission line composite to a new continuum constructed using line-free SED points from the composite of Richards et al. (2006).

Most type-1 objects also required an extra near-infrared component to match the SEDs. This "very hot" component was modeled as a $1000 \mathrm{~K}$ modified blackbody (Equation (3) with $T=1000$ ). The physical origin of this component is unclear, but it is most likely due to hot dust close to the sublimation radius (e.g., Netzer et al. 2007). Gallagher et al. (2007) show that the strength of this component correlates with quasar luminosity. We do not find this to be true for our sample, but note that Gallagher et al. (2007) have a much larger sample size spread over a range of luminosities.

The modeling results for the type- 1 objects are presented in Figure 2, and the results for the type-2 objects are presented in Figure 3. Several differences between the type-1 sample and the type-2 sample are immediately apparent from the fitting results. The type- 1 objects have a strong optical quasar component 


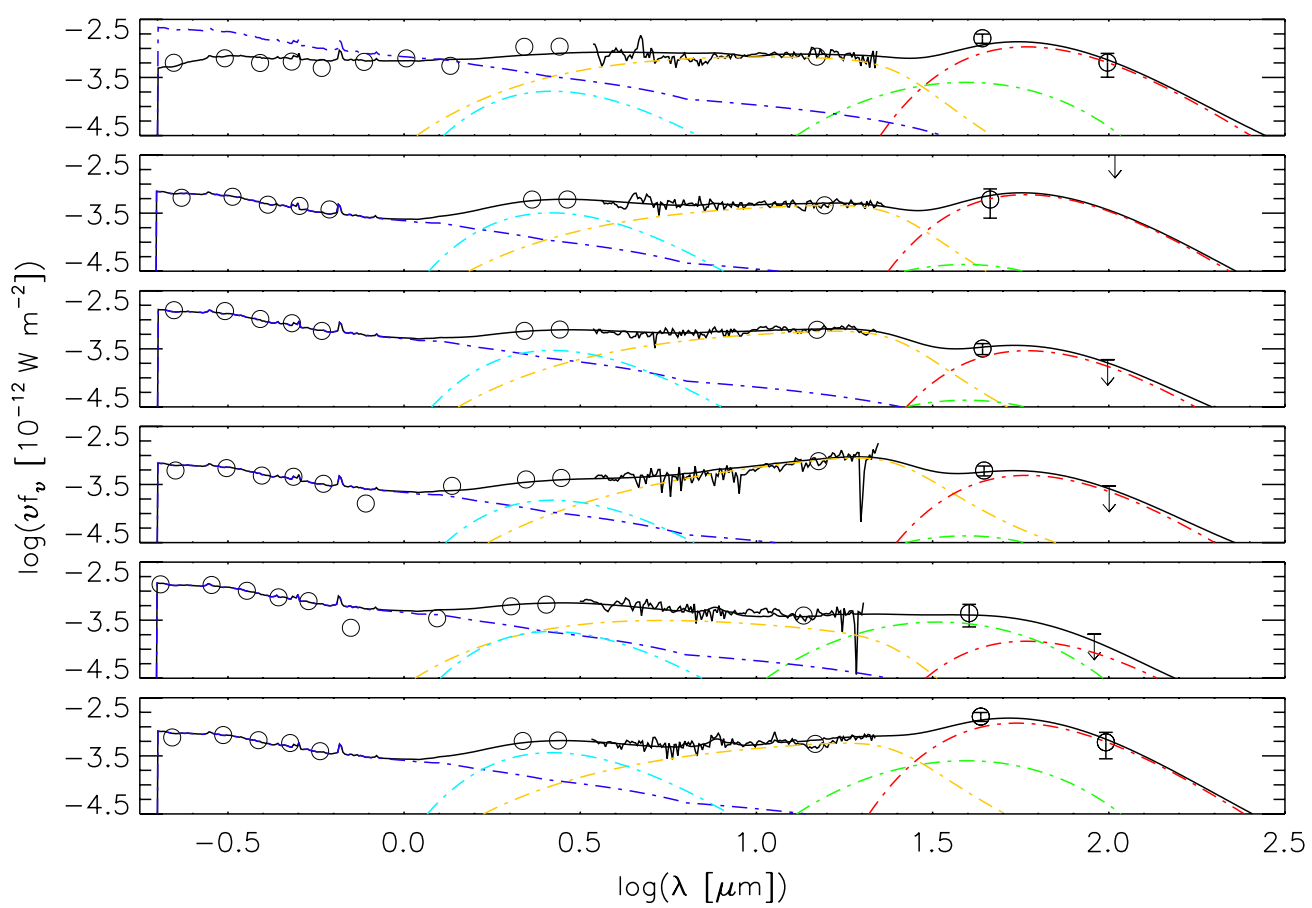

Figure 2. Type-1 sample. The spectra are presented in their rest frame. From top to bottom are SDSS $1711+5841,1711+5855,1713+5950,1717+5930,1717+5948$, and $1718+5849$. The photometry measurements are plotted as circles and the IRS data are plotted as a solid line. We show error bars for the MIPS $70 \mu \mathrm{m}$ and $160 \mu \mathrm{m}$ photometry. Error bars on other photometry measurements are smaller than the plotting symbol. The individual components of the model are plotted as dot-dashed lines and the overall fit as the solid black line. The blue line represents the unreddened quasar composite component, the cyan line the $1000 \mathrm{~K}$ component, the orange line the mid-IR power-law, the green line the warm small grain dust power-law, and the red line the cool $45 \mathrm{~K}$ modified blackbody.

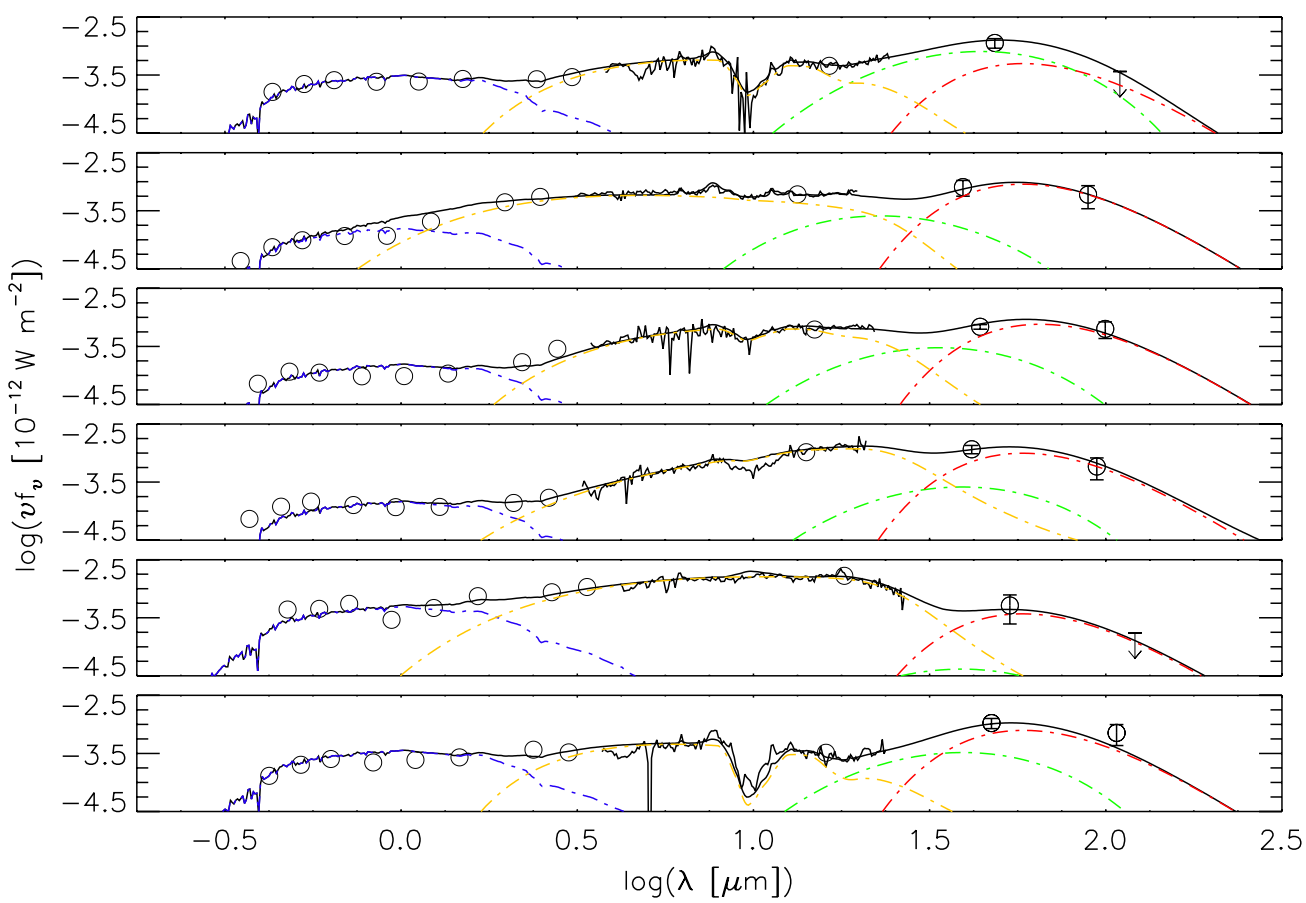

Figure 3. Type- 2 sample. The spectra are presented in their rest frame. From top to bottom are SST $1711+5904,1711+5858,1713+5855,1718+5953,1721+6012$, $1724+5915$. The plotted symbols are the same as in Figure 2 . The photometry measurements are plotted as circles and the IRS data are plotted as a solid line. We show error bars for the MIPS $70 \mu \mathrm{m}$ and $160 \mu \mathrm{m}$ photometry. Error bars on other photometry measurements are smaller than the plotting symbol. The individual components of the model are plotted as dot-dashed lines and the overall fit as the solid black line. The blue line represents the host galaxy stellar component, the orange line the mid-IR power-law, the green line the warm small grain dust power law, and the red line the cool $45 \mathrm{~K}$ modified blackbody.

that can extend significantly into the mid-IR. The $1000 \mathrm{~K}$ component is significant in all six type-1's and also contributes to the mid-IR. Three of the six type-1 object SEDs showed no evidence of the warm small grain dust power-law emission. The type- 2 object SEDs are dominated in the mid-IR by hot dust with no contribution from the optical stellar light and no evidence for the $1000 \mathrm{~K}$ component. Also, present in the mid-IR portion of the type- 2 objects is a variety of PAH emission and silicate absorption. Five of the six type-2 objects have significant contributions to the mid and far-IR from the warm small grain 


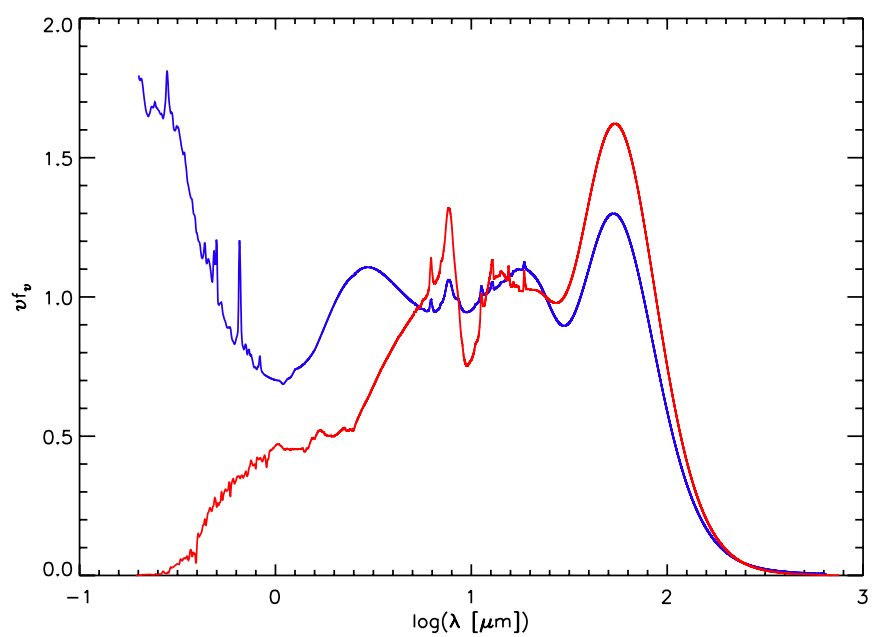

Figure 4. Averaged modeled SEDs of type-1 (blue) and type-2 (red) quasars. The spectra are normalized at $24 \mu \mathrm{m}$. We plot $\nu \mathrm{f}_{v}$ to highlight the differences between the two.

dust power-law. SST $1721+6012$ was the only type-2 not fit well with this component.

We note that the results of the fitting procedure for the object SST 1724+5915 underestimate both the observed amount of PAH emission and the far-IR emission. This is likely due to the large amount of silicate absorption also present in the spectrum of the object. In particular, the $9.7 \mu \mathrm{m}$ absorption feature can affect the shape of the $7.7 \mu \mathrm{m}$ PAH feature, making it difficult to fit with an empirical template. We include the data in our subsequent analysis, but note that star formation rates derived from the far-IR emission and the PAH emission likely underestimate the true star formation of this object (see Section 4.2).

We averaged the modeled type-1 SEDs and type-2 SEDs to highlight the differences between the two samples. We first normalized the SED of each target at $24 \mu \mathrm{m}$ to ensure each object had equal weight. By choosing to normalize at $24 \mu \mathrm{m}$ we can compare the relative amounts of hot and cool dust in the averaged SEDs. Though at $24 \mu \mathrm{m}$, extinction is still present, it is significantly reduced compared to shorter wavelengths. It is clear from Figure 4 that on average type- 2 objects show more PAH emission, more silicate absorption and more far-IR emission than type- 1 objects. In addition, while the very hot $3 \mu \mathrm{m}$ bump is clear in the type- 1 spectrum, no bump is seen in the type- 2 spectrum. We note the caveat that the type- 1 and type- 2 objects could have equivalent amounts of PAH emission if that emission were significantly diluted by a strong mid-IR continuum in type- 1 objects.

The important question is if these observed differences could arise solely due to orientation effects. In that scenario, the optical through mid-IR light is absorbed and re-radiated in the far-IR. To test this, we integrated the optical through farIR fluxes of the normalized and averaged spectra and found them to be identical. These results are consistent with the orientation hypothesis, though the geometry and distribution of the obscuring dust cannot be proved from this simple test.

Furthermore, under the orientation hypothesis, the dust tori of the type- 1 and type- 2 quasars should have the same intrinsic luminosities. Our modeling results show that the tori of the type2 quasars are slightly more luminous than the tori of the type- 1 quasars, though the difference is not statistically significant.

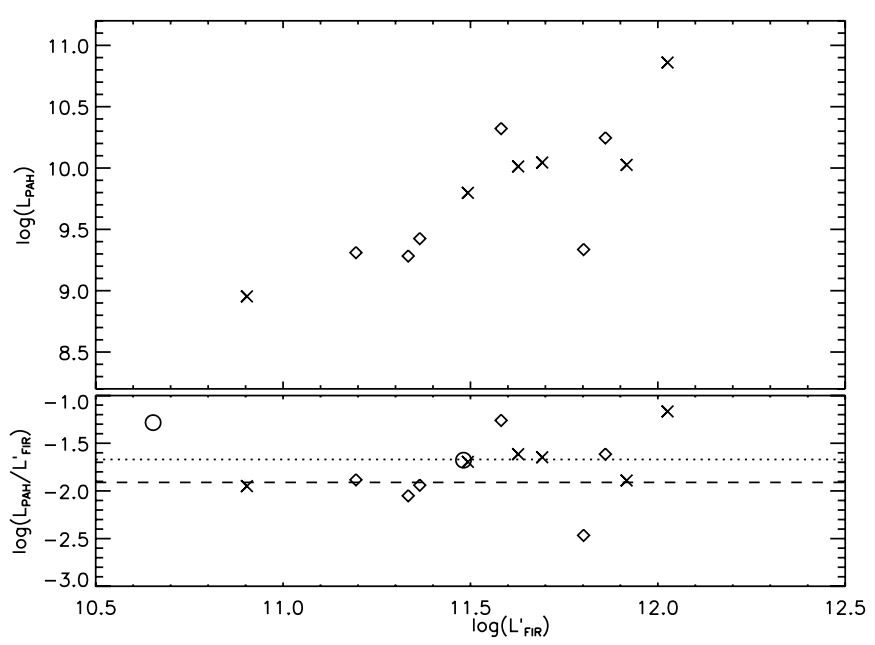

Figure 5. Top: integrated PAH luminosity as measured from our scaled template is plotted against far-IR luminosity. Bottom: Relative amount of PAH emission compared to the far-IR dust emission. In both plots, $L_{\mathrm{FIR}}^{\prime}$ was measured from the warm and cold far-IR components of the model only. $L_{\mathrm{PAH}}$ and $L_{\mathrm{FIR}}^{\prime}$ are in $L_{\odot}$ units. Type- 1 quasars are plotted as diamonds, and type- 2 quasars are plotted as "X"s. The starburst galaxies NGC 7714 and NGC 2623 are plotted as circles (Marshall et al. 2007). The dashed line indicates the median ratio of the type- 1 objects of our sample, and the dotted line indicates the median ratio of the type-2 objects.

This may be a result of our mid-IR selection technique, as those wavelengths are still somewhat affected by extinction. In the next section, we examine the PAH emission and the star formation contribution to the far-IR emission to gain more insight into whether the far-IR emission is different between the two objects.

\subsection{Star Formation Rates}

We characterized and compared the star formation rates of the quasar host galaxies by comparing the PAH emission with the far-IR luminosity of our sample (Figure 5). We measured the far-IR luminosity from only the modeled warm (Equation (2)) and cold dust (Equation (3)) contributions to the SEDs (hereafter $L_{\mathrm{FIR}}^{\prime}$ ), excluding dust heated by the central AGN and emitting in the mid-IR. This definition of $L_{\mathrm{FIR}}^{\prime}$ specifically excludes dust heated by the AGN while still measuring dust heated in star forming regions, even if the two emit at similar or overlapping wavelengths. This is in contrast to using a defined wavelength cutoff, which would measure dust heated by either source. However, it is still important to note that the far-IR emitting dust could also be heated by the AGN if that dust were at large enough distances from the nucleus. It is important to consider this potential AGN contribution to the far-IR emission when calculating SFRs.

The PAH emission is an independent indicator of the star formation activity in the host galaxy. UV and X-ray emission from the AGNs destroy PAHs (Voit 1992), but soft UV emission from star formation excites PAHs into emission. In Figure 5, we find a correlation between PAH emission and far-IR emission similar to previous works (e.g., Schweitzer et al. 2006, MenendezDelmestre et al. 2009). We also normalized the PAH emission to the far-IR emission, so we can compare the relative contribution of star formation to the far-IR emission. We measured the PAH luminosities by integrating our scaled templates, including the full profiles of each individual PAH feature in the template. This is different from many previous works (e.g., Schweitzer et al. 2006, Menendez-Delmestre et al. 2009), where only individual 
Table 3

Luminosities and Star Formation Rates

\begin{tabular}{lcccc}
\hline \multicolumn{1}{c}{ Target } & $\log \left(L_{\mathrm{IR}} / L_{\odot}\right)^{\mathrm{a}}$ & $\log \left(L_{\mathrm{PAH}} / L_{\odot}\right)^{\mathrm{b}}$ & $\mathrm{SFR}\left(M_{\odot} \mathrm{yr}^{-1}\right)^{\mathrm{c}}$ & $\mathrm{SFR}_{\left(M_{\odot} \mathrm{yr}^{-1}\right)^{\mathrm{d}}}$ \\
\hline SDSS 171117.66+584123.8 & 12.25 & 9.3 & 109 & 10 \\
SDSS 171126.94+585544.2 & 11.82 & 9.4 & 40 & 12 \\
SDSS 171334.03+595028.3 & 11.95 & 9.3 & 27 & 10 \\
SDSS 171736.91+593011.5 & 12.05 & 9.3 & 37 & 9 \\
SDSS 171748.43+594820.6 & 12.06 & 10.3 & 66 & 98 \\
SDSS 171818.14+584905.2 & 12.26 & 10.2 & 125 & 82 \\
SST 171106.8+590436 & 11.79 & 10.0 & 73 & 48 \\
SST 171147.4+585839 & 12.46 & 10.9 & 183 & 338 \\
SST 171324.1+585549 & 12.13 & 10.0 & 85 & 52 \\
SST 171831.7+595317 & 12.47 & 10.0 & 143 & 50 \\
SST 172123.1+601214 & 11.48 & 9.0 & 14 & 4 \\
SST 172458.3+591545 & 11.77 & 9.8 & 54 & 29 \\
\hline
\end{tabular}

Notes.

a The luminosity $L_{\mathrm{IR}}$ corresponds to the integrated modeled SED from 8to $1000 \mu \mathrm{m}$.

b The PAH luminosity is measured from the integrated profiles of all PAH features in the model.

${ }^{c}$ Star formation rates derived from the sum of the warm and cold dust components of the model only. The SFR was calculated using the Kennicutt (1998) relation.

${ }^{\mathrm{d}}$ Star formation rates derived from PAH luminosity using the results of Marshall et al. (2007) to convert from $L_{\mathrm{PAH}}$ to $L_{\text {FIR }}$ and the Kennicutt (1998) relation.

e The fit of SST 1724+5915 underestimates the PAH luminosity and far-IR emission, thus also underestimating the SFRs derived from these quantities.

PAH features are measured, and definite wavelength cutoffs are applied. We find that the quasars as a whole have a fairly constant ratio across a range of far-IR luminosities. The median $L_{\mathrm{PAH}} / L_{\mathrm{FIR}}^{\prime}$ ratio for the type- 1 objects of our sample is 0.012 , and the median for the type- 2 objects is 0.021 . We note that the difference between the type- 1 objects and type- 2 objects is not significant considering the scatter in the sample. The median of our overall sample is 0.017. In comparison, Marshall et al. (2007) measure PAH luminosity using a similar method to ours and find that the starburst galaxies NGC 7714 and NGC 2623 have $L_{\mathrm{PAH}} / L_{\mathrm{FIR}}$ ratios of 0.052 and 0.021 , respectively. We cannot draw strong conclusions based on measurements of two objects, and note that many objects of our sample have ratios consistent with these objects. However, the average $L_{\mathrm{PAH}} / L_{\mathrm{FIR}}$ from Marshall et al. is 0.037, higher than the median value of our sample. If taken at face value, these numbers indicate that some of the cool far-IR emitting dust is heated by AGN emission instead of stellar emission.

In Table 3, we present the integrated mid- to far-IR emission (8-1000 $\mu \mathrm{m}$, hereafter $\left.L_{\mathrm{IR}}\right)$. We find that nearly all of our targets have $\log \left(L_{\mathrm{IR}} / L_{\odot}\right) \geqslant 11.75$. SST $1721+6012$ is the only object with $\log \left(L_{\mathrm{IR}} / L_{\odot}\right)<11.75$. We also list the SFRs of our sample in Table 3. We calculated SFRs in two different ways. First, we converted the integrated warm and cold dust components of our model into SFRs using the Kennicutt (1998) calibration for starburst galaxies:

$$
\operatorname{SFR}\left(M_{\odot} \mathrm{yr}^{-1}\right)=4.5 \times 10^{-44} L_{\mathrm{FIR}}^{\prime}\left(\mathrm{erg} \mathrm{s}^{-1}\right) .
$$

Although our objects have IR luminosities similar to those of ULIRGs, their SFRs are lower than those of typical ULIRGs. This is because some of the IR luminosity of our objects is due to AGN-heated dust, and we explicitly exclude this component when calculating SFRs. The SFRs of our sample are instead typical of LIRGs, which can range from 20-200 $M_{\odot} \mathrm{yr}^{-1}$ (e.g., Sanders et al. 2003). We find that the type- 2 objects show higher SFRs on average $\left(92 M_{\odot} \mathrm{yr}^{-1}\right)$ than the type-1 objects $\left(67 M_{\odot} \mathrm{yr}^{-1}\right)$. However, the scatter is large, and the object with the lowest SFR is a type-2, SST $1721+6012$. We also independently calculated the SFR from the PAH emission. This can be done by predicting a far-IR luminosity based on the PAH luminosity. We integrated our scaled PAH template to measure the PAH luminosity of each object in our sample. We adopt an average ratio of $L_{\mathrm{PAH}} / L_{\mathrm{FIR}} \sim 0.037$ based on the results of Marshall et al. (2007). Subsequently, for all objects in our sample except two, the SFR predicted from the PAH luminosity is lower than the SFR predicted by integrating the warm and cold dust components of the far-IR $\left(L_{\mathrm{FIR}}^{\prime}\right)$. Again we found that the average type- 2 quasar has a higher SFR $\left(87 M_{\odot} \mathrm{yr}^{-1}\right)$ than the average type- 1 quasar $\left(37 M_{\odot} \mathrm{yr}^{-1}\right)$.

\section{SUMMARY AND DISCUSSION}

We obtained mid-IR spectra and far-IR photometry for six type-1 and six type- 2 mid-IR selected quasars. We modeled the SEDs of our sample from the optical to the far-IR using available SDSS, 2MASS, and IRAC photometry, in addition to our IRS spectra and MIPS photometry. We compared the modeled SEDs of the quasars and calculated SFRs based on PAH and far-IR emission. Our main results are the following.

1. The type-1 quasar mid-IR spectra show a featureless continuum with significant $3 \mu \mathrm{m}$ emission. The full optical through far-IR SEDs are typically fit well with an optical quasar component, a $1000 \mathrm{~K}$ modified blackbody, a mid-IR power-law $(\sim 3-30 \mu \mathrm{m})$, and a cool dust far-IR modified blackbody $(45 \mathrm{~K})$. Three of the objects also show evidence of warm small grain dust fit with a power-law ( 25-65 $\mu \mathrm{m})$.

2. The type-2 quasar mid-IR spectra show a variety of PAH emission and silicate absorption strengths. The full optical through far-IR SEDs are fit well using a stellar population in the optical, a mid-IR power-law $(\sim 3-30 \mu \mathrm{m})$, varying amounts of a PAH emission template and silicate absorption in the mid-IR, warm small grain power-law emission $(\sim 25$ $65 \mu \mathrm{m})$ as well as a cool dust modified blackbody ( $45 \mathrm{~K})$.

3 . Our averaged and normalized modeled spectra show that type-2 quasars exhibit more far-IR emission than type-1 quasars. The excess is roughly equivalent to the deficit in 
the optical with respect to the type-1 quasars, which is consistent with the orientation hypothesis. In this scenario the increased far-IR emission arises from obscuring dust that is reradiating absorbed light.

4. We measured $L_{\mathrm{PAH}}$ by integrating our scaled template for each object. We compared the typical $L_{\mathrm{PAH}}$ to $L_{\mathrm{FIR}}^{\prime}$ ratio of our sample to that of starburst galaxies that have had $L_{\mathrm{PAH}}$ measured in a similar manner as the method we used. Within our sample, we found that the type-2 objects have a larger ratio than the type- 1 objects. We note that the difference between the two is not significant. Considering our overall sample, we found that the ratio is smaller than for the starburst galaxies (Marshall et al. 2007). This result suggests that some far-IR emitting dust can be warmed by the central AGNs rather than by young stars. However, a more direct comparison between AGNs and starburst galaxies using larger samples would be needed to further quantify the amount of AGN-heated far-IR emitting dust.

5. We calculated the SFRs of our sample using two methods. First we integrated the warm small grain and cool modified blackbody emission (excluding all other components) of the modeled SEDs and used the Kennicutt (1998) relation to calculate the SFRs. We also converted the $L_{\mathrm{PAH}}$ to $L_{\mathrm{FIR}}$ (Marshall et al. 2007) to measure the SFRs based on the PAH emission only. In each case the type- 2 objects had higher SFR on average than the type-1 objects. However, the difference is not significant given the scatter.

6. We integrated our modeled SEDs from 8 to $1000 \mu \mathrm{m}$ and found that seven of the 12 can be considered ULIRGs $\left(\log \left(L_{\mathrm{IR}} / L_{\odot}\right) \geqslant 12\right)$, while four others have $\log \left(L_{\mathrm{IR}} / L_{\odot}\right) \geqslant 11.75$. The sole exception is SST $1721+6012$, which has $\log \left(L_{\mathrm{IR}} / L_{\odot}\right)=11.48$. While our sample appears to have IR luminosities similar to ULIRGs, the quasars exhibit SFRs more typical of LIRGs. We found the AGN can heat most of the dust in the mid-IR and even some in the far-IR. Thus measuring SFR in quasars from the full 8-1000 $\mu \mathrm{m}$ SED overestimates the amount of dust warmed by young stars and the true SFR.

7. The modeling results suggest that type-1 quasars can have mid-IR fluxes that have significant contributions from the optical power-law and $3 \mu \mathrm{m}$ bump. This $3 \mu \mathrm{m}$ component is not required for the type-2 SEDs, and the hot dust most likely originates near the sublimation radius of the AGNs. If this component is present in the type-2 quasars, it must be at least partially obscured by cooler dust, either a dusty torus or dust in the host galaxy. Lacy et al. (2007) showed that the host galaxies of the type- 2 quasars have a range of inclinations. Therefore, the obscuration of the $3 \mu \mathrm{m}$ bump, if it is intrinsically present, should be from material close to the nucleus and independent of galaxy orientation.

While our analysis does not distinguish definitively between orientation and evolution based unification schemes, it does directly address the dust content, ongoing star formation in the host galaxies and the AGN contribution to the far-IR emission. Our results are consistent with the orientation hypothesis, but we cannot rule out an evolutionary connection. The star formation based on PAH measurements is a larger contributor to the far-IR luminosity than the AGN for type-2 objects compared to type-1 objects. This difference is only suggestive due to the scatter of both $L_{\mathrm{PAH}}$ and $L_{\mathrm{FIR}}^{\prime}$ in our sample and could arise from intrinsic differences in the bolometric luminosities of the objects. The $L_{\mathrm{PAH}} / L_{\mathrm{FIR}}^{\prime}$ ratio in both type-1 and type-2 quasars is lower than that of starburst galaxies, suggesting that some emission in the far-IR is from dust heated by the AGNs and not star formation. This is an area for continued research, as our analysis was based on few objects.

We thank the anonymous referee for useful comments and suggestions that helped improve both the paper's content and presentation. This work is based on observations made with the Spitzer Space Telescope, which is operated by the Jet Propulsion Laboratory, California Institute of Technology, under a contract with NASA. This research has made use of the NASA/ IPAC Extragalactic Database (NED) which is operated by the Jet Propulsion Laboratory, California Institute of Technology, under contract with the National Aeronautics and Space Administration. Support for this work was provided by NASA through an award issued by J.P.L. Additional support was provided by the National Science Foundation, under grant number AST 0507450.

\section{Facilities: Spitzer (IRS); Spitzer (MIPS)}

\section{REFERENCES}

Antonucci, R. 1993, ARA\&A, 31, 473

Bennert, N., Canalizo, G., Jungwiert, B., Stockton, A., Schweizer, F., Peng, C., \& Lacy, M. 2008, ApJ, 677, 846

Brandl, B. R., et al. 2006, ApJ, 653, 1129

Bruzual, G., \& Charlot, S. 2003, MNRAS, 344, 1000

Canalizo, G., Bennert, N., Jungwiert, B., Stockton, A., Schweizer, F., Lacy, M., \& Peng, C. 2007, ApJ, 669, 801

Chiar, J. E., \& Tielens, A. G. G. M. 2006, ApJ, 637, 774

Croom, S., et al. 2004, MNRAS, 349, 1397

Dunlop, J. S., et al. 2003, MNRAS, 340, 1095

Fadda, D., et al. 2006, AJ, 131, 2859

Frayer, D. T., et al. 2006, AJ, 131, 250

Gallagher, S. C., Richards, G. T., Lacy, M., Hines, D. C., Elitzur, M., \& StorrieLombardi, L. J. 2007, ApJ, 661, 30

Hao, L., Weedman, D. W., Spoon, H. W. W., Marshall, J. A., Levenson, N. A., Elitzur, M., \& Houck, J. R. 2007, ApJ, 655, L77

Kennicutt, R. C. 1998, ARA\&A, 36, 189

Lacy, M., Sajina, A., Petric, A. O., Seymour, N., Canalizo, G., Ridgway, S. E., Armus, L., \& Storrie-Lombardi, L. J. 2007, ApJ, 669, L61

Lacy, M., et al. 2004, ApJS, 154, 166

Lacy, M., et al. 2005, ApJS, 161, 41

Lutz, D., et al. 2008, ApJ, 684, 853

Marshall, J. A., Herter, T. L., Armus, L., Charmandaris, V., Spoon, H. W. W., Bernard-Salas, J., \& Houck, J. R. 2007, ApJ, 670, 129

Menendez-Delmestre, K., et al. 2009, ApJ, 699, 667

Netzer, H., et al. 2007, ApJ, 666, 806

Oke, J. B. 1990, AJ, 99, 1621

Richards, G. T., et al. 2006, ApJS, 166, 470

Sanders, D. B., Mazzarella, J. M., Kim, D. C., Surace, J. A., \& Soifer, B. T. 2003, AJ, 126, 1607

Sanders, D. B., et al. 1988, ApJ, 325, 74

Sajina, A., Scott, D., Dennefeld, D., Dole, H., Lacy, M., \& Lagache, G. 2006, MNRAS, 369, 939

Sajina, A., Yan, L., Armus, L., Choi, P., Fadda, D., Helou, G., \& Spoon, H. 2007, ApJ, 664, 713

Schneider, D. P., et al. 2003, AJ, 126, 2579

Schweitzer, M., et al. 2006, ApJ, 649, 79

Spoon, H. W. W., Marshall, J. A., Houck, J. R., Elitzur, M., Hao, L., Armus, L., Brandl, B. R., \& Charmandaris, V. 2007, ApJ, 654, 49

Vanden Berk, D. E., et al. 2001, AJ, 122, 549

Voit, M. 1992, MNRAS, 258, 841

Wolf, C., et al. 2003, A\&A, 408, 499

Zakamska, N. L., Gomez, L., Strauss, M. A., \& Krolik, J. H. 2008, AJ, 136, 1607

Zakamska, N. L., et al. 2005, AJ, 129, 1212 